\title{
Bayesian Compressive Sensing Using Normal Product Priors
}

\author{
Zhou Zhou, Kaihui Liu, and Jun Fang, Member, IEEE
}

\begin{abstract}
In this paper, we introduce a new sparsitypromoting prior, namely, the "normal product" prior, and develop an efficient algorithm for sparse signal recovery under the Bayesian framework. The normal product distribution is the distribution of a product of two normally distributed variables with zero means and possibly different variances. Like other sparsityencouraging distributions such as the Student's $t$-distribution, the normal product distribution has a sharp peak at origin, which makes it a suitable prior to encourage sparse solutions. A twostage normal product-based hierarchical model is proposed. We resort to the variational Bayesian (VB) method to perform the inference. Simulations are conducted to illustrate the effectiveness of our proposed algorithm as compared with other state-of-theart compressed sensing algorithms.
\end{abstract}

Index Terms-Compressed Sensing, sparse Bayesian learning, normal product prior.

\section{INTRODUCTION}

C OMPRESSED sensing [1], [2] is a new data acquisition technique that has attracted much attention over the past decade. Existing methods for compressed sensing can generally be classified into the following categories, i.e. the greedy pursuit approach [3], the convex relaxation-type approach [4] and the nonconvex optimization method [5]. Another class of compressed sensing techniques that have received increasing attention are Bayesian methods. In the Bayesian framework, the signal is usually assigned a sparsity-encouraging prior, such as the Laplace prior and the Gaussian-inverse Gamma prior [6], to encourage sparse solutions. It has been shown in a series of experiments that Bayesian compressed sensing techniques [7] demonstrate superiority for sparse signal recovery as compared with the greedy methods and the basis pursuit method. One of the most popular prior model for Bayesian compressed sensing is the Gaussian-inverse Gamma prior proposed in [6]. The Gaussian-inverse Gamma prior is a two-layer hierarchical model in which the first layer specifies a Gaussian prior to the sparse signal and an inverse Gamma priori is assigned to the parameters characterizing the Gaussian prior. As discussed in [8], this two-stage hierarchical model is equivalent to imposing a Student's $t$-distribution on the sparse signal. Besides the Gaussian-inverse Gamma prior, authors in [9] employ Laplace priors for the sparse signal to promote sparse solutions.

Zhou Zhou, Kaihui Liu, and Jun Fang are with the National Key Laboratory on Communications, University of Electronic Science and Technology of China, Chengdu 611731, China, Email: ZhouZhou@std.uestc.edu.cn; kaihuil@std.uestc.edu.cn; JunFang@uestc.edu.cn

This work was supported in part by the National Science Foundation of China under Grant 61172114.
In this paper, we introduce a new sparsity-encouraging prior, namely, the normal product (NP) prior, for sparse signal recovery. A two-stage normal product-based hierarchical model is established, and we resort to the variational Bayesian (VB) method to perform the inference for the hierarchical model. Our experiments show that the proposed algorithm achieves similar performance as the sparse Bayesian learning method, while with less computational complexity.

\section{THE BAYESIAN NETWORK}

In the context of compressed sensing, we are given a noise corrupted linear measurements of a vector $\mathbf{x}_{0}$

$$
\mathbf{y}=\mathbf{A} \mathbf{x}_{0}+\mathbf{n}
$$

Here $\mathbf{A} \in \mathbf{R}^{M \times N}$ is an underdetermined measurement matrix with low coherence of columns, $\mathbf{n} \in \mathbf{R}^{M}$ represents the acquisition noise vector and $\mathbf{x}_{0}$ a sparse signal. For the inverse problem, both $\mathbf{x}_{0}$ and $\mathbf{n}$ are unknown, and our goal is to recover $\mathbf{x}_{0}$ from $\mathbf{y}$. We formulate the observation noise as zero mean independent Gaussian variable with variance $\xi^{2}$ i.e. $\mathbf{y} \sim$ $N\left(\mathbf{A x}_{0}, \xi^{2} \mathbf{I}\right)$ and seek a sparse signal $\mathbf{x} \triangleq\left(x_{1}, x_{2}, \ldots x_{N}\right)$ for $\mathrm{x}_{0}$.

In this section, we utilize a two-stage hierarchical bayesian prior for the signal model. In the first layer of signal model, we use the Normal Product (NP) distribution as sparseness prior:

$$
\mathbf{x} \sim N P(\mathbf{0}, \boldsymbol{\Sigma})
$$

where $\mathbf{0} \in \mathbf{R}^{N}$ stands for its mean and $\boldsymbol{\Sigma}$ given as a diagonal matrix $\operatorname{diag}\left({\sigma_{1}}^{2}, \sigma_{2}{ }^{2}, \ldots, \sigma_{N}{ }^{2}\right) \in \mathbf{R}^{N \times N}$ represents its variance. For each element $x_{i}$ of $\mathbf{x}$, its probability density distribution is $K_{0}\left(\frac{\left|x_{i}\right|}{\sigma_{i}}\right) / \pi \sigma_{i}$, which exhibits a sharp peak

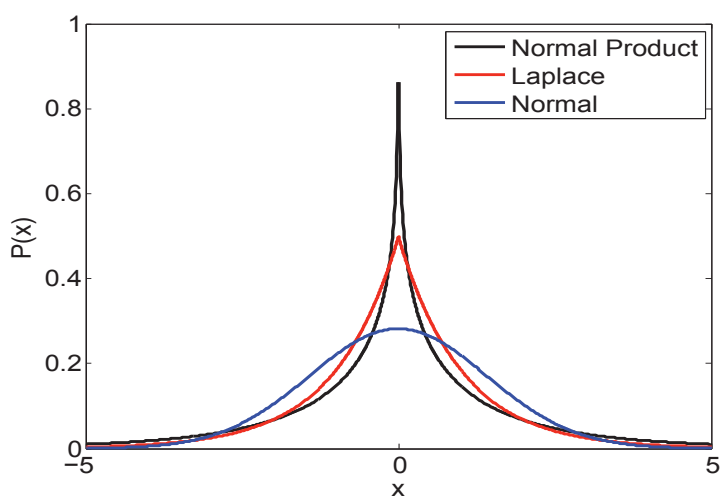

Fig. 1. Three kinds of PDF with the same standard deviation 
at the origin and heavy tails, where $K_{0}(\cdot)$ is the zero order modified Bessel function of the second kind [10] thus the probability density function (PDF) of $\mathrm{x}$ is

$$
\frac{1}{\pi^{N}} \prod_{i=1}^{N} \frac{1}{\sigma_{i}} K_{0}\left(\frac{\left|x_{i}\right|}{\sigma_{i}}\right) .
$$

A NP distributed scaler variate $x_{i} \in \mathbf{R}$ can be decomposed as the product of two independent normally distributed variables i.e. if $x_{i} \sim N P\left(0, \sigma_{i}^{2}\right)$, then there are $a_{i} \sim N\left(0, \kappa_{i}^{2}\right)$ and $b_{i} \sim N\left(0, \gamma_{i}^{2}\right)$ satisfying $x_{i}=a_{i} b_{i}$ with moment relationship $\sigma_{i}=\gamma_{i} \kappa_{i}[11]$. We call this property as the generating rule of Normal Product distribution. Similarly for the vector $\mathbf{x}$, we can decompose it into the Hadamard product of two independent virtual normally distributed vector variables $\mathbf{a}$ and $\mathbf{b}$ whose variance are diagonal matrix $\kappa^{2} \triangleq \operatorname{diag}\left(\kappa_{1}^{2}, \kappa_{2}^{2}, \ldots, \kappa_{N}^{2}\right)$ and $\gamma^{2} \triangleq \operatorname{diag}\left(\gamma_{1}^{2}, \gamma_{2}^{2}, \ldots, \gamma_{N}^{2}\right)$ respectively i.e. $\boldsymbol{x}=\boldsymbol{a} \circ \boldsymbol{b}$ and $\boldsymbol{\Sigma}=\boldsymbol{\kappa}^{2} \circ \boldsymbol{\gamma}^{2}$, where $\circ$ denote the Hadamard product. Finally, we set $\sigma_{i}^{-2}$ as a realization of Gamma hyperprior $\Gamma(\alpha, \beta)$ and choose $\alpha \rightarrow 0, \beta \rightarrow 0$ to construct a sharper distribution of $\mathbf{x}$ during the variational procedure [12] for the second layer of signal.

According to the generating rule of Normal Product mentioned above, we can add two parallel nodes $\mathbf{a}$ and $\mathbf{b}$ to construct one latent layer. Then the posterior distribution can be expressed as:

$$
P_{\mathbf{x}}(\mathbf{x}, \mathbf{\Sigma} \mid \mathbf{y} ; \alpha, \beta)=\int P(\mathbf{x}, \mathbf{a}, \mathbf{b}, \mathbf{\Sigma} \mid \mathbf{y} ; \alpha, \beta) d \mathbf{a} d \mathbf{b}
$$

where

$$
\begin{aligned}
& P(\mathbf{x}, \mathbf{a}, \mathbf{b}, \mathbf{\Sigma} \mid \mathbf{y} ; \alpha, \beta) \\
& \propto P(\mathbf{y} \mid \mathbf{x}) P(\mathbf{x} \mid \mathbf{a}, \mathbf{b}) P\left(\mathbf{a} \mid \kappa^{2}\right) P\left(\mathbf{b} \mid \gamma^{2}\right) P(\mathbf{\Sigma} \mid \alpha, \beta) \\
& =P(\mathbf{y}-\mathbf{A x}) \delta(\mathbf{x}-\mathbf{a} \circ \mathbf{b}) P\left(\mathbf{a} \mid \kappa^{2}\right) P\left(\mathbf{b} \mid \gamma^{2}\right) P(\mathbf{\Sigma} \mid \alpha, \beta)
\end{aligned}
$$

and $\delta(\cdot)$ denotes the Dirac Delta function. In this expression we know that the value of $\mathbf{x}$ must be equal to $\mathbf{a} \circ \mathbf{b}$ while keeping the value of $P(\mathbf{x}, \mathbf{a}, \mathbf{b}, \mathbf{\Sigma} \mid \mathbf{y} ; \alpha, \beta)$ nonzero. Consider the Bayesian Risk function:

$$
\begin{aligned}
& E(L(\hat{\mathbf{x}}, \mathbf{x}))=\int L(\hat{\mathbf{x}}, \mathbf{x}) P_{\mathbf{x}}(\mathbf{x}, \boldsymbol{\Sigma} \mid \mathbf{y} ; \alpha, \beta) d \mathbf{x} d \mathbf{\Sigma} \\
& \propto \int L(\hat{\mathbf{x}}, \mathbf{a} \circ \mathbf{b}) P_{\mathbf{a}, \mathbf{b}}(\mathbf{a}, \mathbf{b}, \boldsymbol{\Sigma} \mid \mathbf{y} ; \alpha, \beta) d \mathbf{a} d \mathbf{b} d \mathbf{\Sigma}
\end{aligned}
$$

where

$$
\begin{aligned}
& P_{\mathbf{a}, \mathbf{b}}(\mathbf{a}, \mathbf{b}, \mathbf{\Sigma} \mid \mathbf{y} ; \alpha, \beta) \\
& =P(\mathbf{y}-\mathbf{A}(\mathbf{a} \circ \mathbf{b})) P\left(\mathbf{a} \mid \boldsymbol{\kappa}^{2}\right) P\left(\mathbf{b} \mid \gamma^{2}\right) P(\boldsymbol{\Sigma} \mid \alpha, \beta)
\end{aligned}
$$

and $L(\hat{\mathbf{x}}, \mathbf{x})$ represents the loss function. Thus, we can replace $P_{\mathbf{x}}(\mathbf{x}, \boldsymbol{\Sigma} \mid \mathbf{y} ; \alpha, \beta)$ with $P_{\mathbf{a}, \mathbf{b}}(\mathbf{a}, \mathbf{b}, \boldsymbol{\Sigma} \mid \mathbf{y} ; \alpha, \beta)$ in the inference procedure while maintaining the value of the Bayesian risk function at a consistent level. So the modified Bayesian Network is:

$$
\begin{gathered}
\mathbf{y} \sim N\left(\mathbf{A}(\mathbf{a} \circ \mathbf{b}), \xi^{2} \mathbf{I}\right) \\
\mathbf{a} \sim N\left(\mathbf{0}, \boldsymbol{\kappa}^{2}\right) \\
\mathbf{b} \sim N\left(\mathbf{0}, \gamma^{2}\right) \\
\sigma_{i}^{-2}=\gamma_{i}^{-2} \kappa_{i}^{-2} \sim \Gamma(\alpha, \beta)
\end{gathered}
$$

as depicted in Fig. 2

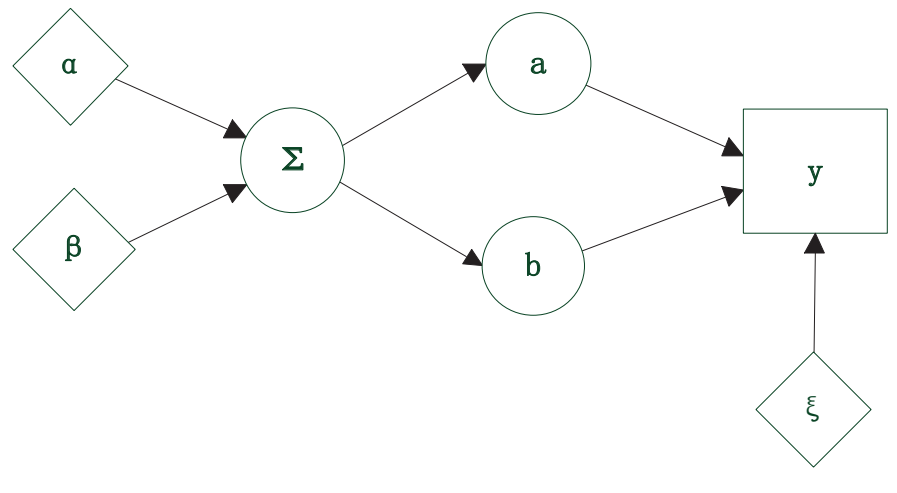

Fig. 2. The Bayesian Network

\section{The VARATIONAL BAyEsian INFERENCE}

In order to infer the bayesian network, we use the mean-field variational Bayesian method to analytically approximate the entire posterior distribution of latent variable. In this theorem, it was assumed that the approximate variational distribution $q(\mathbf{z} \mid \mathbf{y})$ where $\mathbf{z}$ stands for the latent variable in the model could be factorized into the product $\prod_{i=1}^{l} q_{i}\left(\mathbf{z}_{i} \mid \mathbf{y}\right)$ for the partition $\mathbf{z}_{1}, \ldots, \mathbf{z}_{l}$ of $\mathbf{z}$. It could be shown that the solution of the variational method for each factor $q_{i}\left(\mathbf{z}_{i} \mid \mathbf{y}\right)$ could be written as:

$$
q_{i}{ }^{*}\left(\mathbf{z}_{i} \mid \mathbf{y}\right)=\frac{e^{<\ln p(\mathbf{z} \mid \mathbf{y}))>\neq \mathbf{z}_{i}}}{\int e^{<\ln p(\mathbf{z} \mid \mathbf{y}))>\neq \mathbf{z}_{i}} d \mathbf{z}_{i}}
$$

where $<\ln p(\mathbf{z} \mid \mathbf{y}))>_{\neq \mathbf{z}_{i}}$ means taking the expectation over the set of $\mathbf{z}$ using the variational distribution except $\mathbf{z}_{i}$ [13] . Applying the variational Bayesian method upon the bayesian model mentioned in secetion [I], the posterior approximation of $\boldsymbol{\Sigma}, \mathbf{a}, \mathbf{b}$ are respectively:

$$
\begin{aligned}
& \ln \left(q^{*}(\mathbf{a})\right) \propto<\ln P(\mathbf{a}, \mathbf{b}, \boldsymbol{\Sigma} \mid \mathbf{y} ; \alpha, \beta)>_{q^{*}(\mathbf{b}), q^{*}(\boldsymbol{\Sigma})} \\
& \ln \left(q^{*}(\mathbf{b})\right) \propto<\ln P(\mathbf{a}, \mathbf{b}, \boldsymbol{\Sigma} \mid \mathbf{y} ; \alpha, \beta)>_{q^{*}(\mathbf{a}), q^{*}(\boldsymbol{\Sigma})} \\
& \ln \left(q^{*}(\boldsymbol{\Sigma})\right) \propto<\ln P(\mathbf{a}, \mathbf{b}, \boldsymbol{\Sigma} \mid \mathbf{y} ; \alpha, \beta)>_{q^{*}(\mathbf{b}), q^{*}(\mathbf{a})}
\end{aligned}
$$

\section{A. posterior approximation of $\mathbf{a}$ and $\mathbf{b}$}

Substituting (3), (4), (5) and (6) into (8), we can arrive at:

$$
q^{*}(\mathbf{b}) \sim N\left(\boldsymbol{\Gamma}^{-1} \mathbf{c}^{\prime}, \boldsymbol{\Gamma}^{\prime-1}\right)
$$

where

$$
\boldsymbol{\Gamma}^{\prime}=\frac{1}{\xi^{2}}\left(\mathbf{A}^{T} \mathbf{A}\right) \circ<\mathbf{a a}^{T}>_{q^{*}(\mathbf{a})}+<\boldsymbol{\gamma}^{-2}>_{q^{*}(\boldsymbol{\gamma})}
$$

and

$$
\mathbf{c}^{\prime}=\frac{1}{\xi^{2}} \operatorname{diag}\left(<\mathbf{a}>_{q^{*}(\mathbf{a})}\right) \mathbf{A}^{T} \boldsymbol{y}
$$

. From the principle of variational inference, we know that $q(\boldsymbol{b})$ is an approximation of $P(\boldsymbol{b} \mid \boldsymbol{y})$ i.e. $P(\boldsymbol{b} \mid \boldsymbol{y}) \approx q(\mathbf{b})$ and in order to get a more concise iteration formula, we choose the relaxation of the secondary moment as:

$$
\begin{gathered}
<\mathbf{a a}^{T}>_{q^{*}(a)} \approx<\mathbf{a}>_{q^{* *}(a)}<\mathbf{a}^{T}>_{q^{* *}(a)} \\
<\boldsymbol{\gamma}^{-2}>_{q^{*}(\boldsymbol{\gamma})} \approx<\boldsymbol{\gamma}>_{q^{* *}(\boldsymbol{\gamma})}^{-2}
\end{gathered}
$$


This relaxation can be interpreted as ignoring the posteriori variance during the updating, which is inspired by the fact that after the learning procedure the posteriori variance always approaches zero to ensure the posteriori mean's concentration on the estimated value.

Then the corrected posterior approximation is $q^{* *}(\mathbf{b}) \sim$ $N\left(\boldsymbol{\Gamma}^{-1} \mathbf{c}, \boldsymbol{\Gamma}^{-1}\right)$ where

$$
\begin{aligned}
& \boldsymbol{\Gamma}^{-1}=\left\{\frac{1}{\xi^{2}}\left(\mathbf{A d i a g}\left(<\mathbf{a}>_{q^{* *}(\mathbf{a})}\right)\right)^{T} \mathbf{A} \operatorname{diag}\left(<\mathbf{a}>_{q^{* *}(\mathbf{a})}\right)\right. \\
& +<\boldsymbol{\gamma}>_{\left.q^{* *}(\boldsymbol{\gamma})^{-2}\right\}^{-1}}
\end{aligned}
$$

and

$$
\mathbf{c}=\frac{1}{\xi^{2}} \operatorname{diag}\left(<\mathbf{a}>_{q^{* *}(\mathbf{a})}\right) \mathbf{A}^{T} \mathbf{y}
$$

In the noise free case, using Woodbury identity we have:

$$
\begin{aligned}
& \lim _{\xi \rightarrow 0} \boldsymbol{\Gamma}^{-1} \mathbf{c} \\
& =<\boldsymbol{\gamma}>_{q^{* *}(\boldsymbol{\gamma})}\left(\mathbf{A} \operatorname{diag}\left(<\mathbf{a}>_{q^{* *}(\mathbf{a})}\right)<\boldsymbol{\gamma}>_{q^{* *}(\boldsymbol{\gamma})}\right)^{+} \mathbf{y}
\end{aligned}
$$

and

$$
\begin{aligned}
& \lim _{\xi \rightarrow 0} \boldsymbol{\Gamma}^{-1} \\
& =\left(\mathbf{I}-<\boldsymbol{\gamma}>_{\mathbf{q}^{* *}(\boldsymbol{\gamma})}\left(\operatorname{Adiag}\left(<\mathbf{b}>_{\mathbf{q}^{* *}(\mathbf{b})}\right)<\boldsymbol{\gamma}>_{\mathbf{q}^{* *}(\boldsymbol{\gamma})}\right)^{+}\right) \\
& \times \mathbf{A d i a g}\left(<\mathbf{a}>_{q^{* *}(\mathbf{a})}\right)<\boldsymbol{\gamma}>_{q^{* *}(\boldsymbol{\gamma})}^{2}
\end{aligned}
$$

Similarly, for a, substituting (3), (4), (5) and (6) into (7), we have:

$$
q^{*}(\mathbf{a}) \sim N\left(\mathbf{H}^{\prime-1} \mathbf{f}^{\prime}, \mathbf{H}^{\prime-1}\right)
$$

where

$$
\mathbf{H}^{\prime}=\frac{1}{\xi^{2}}\left(\mathbf{A}^{T} \mathbf{A}\right) \circ<\mathbf{b b}^{T}>_{q^{*}(\mathbf{b})}+<\boldsymbol{\kappa}^{-2}>_{q^{*}(\boldsymbol{\kappa})}
$$

and

$$
\mathbf{f}^{\prime}=\frac{1}{\xi^{2}} \operatorname{diag}\left(<\mathbf{b}>_{q^{*}(\mathbf{b})}\right) \mathbf{A}^{T} \mathbf{y}
$$

So the corrected approximation is:

$$
q^{* *}(\mathbf{a}) \sim N\left(\mathbf{H}^{-1} \mathbf{f}, \mathbf{H}^{-1}\right)
$$

Then again, in the noiseless case, we have

$$
\begin{aligned}
& \lim _{\xi \rightarrow 0} \mathbf{H}^{-1} \mathbf{f} \\
& =<\boldsymbol{\kappa}>_{q^{* *}(\boldsymbol{\kappa})}\left(\mathbf{A d i a g}\left(<\mathbf{b}>_{q^{* *}(\mathbf{b})}\right)<\boldsymbol{\kappa}>_{q^{* *}(\boldsymbol{\kappa})}\right)^{+} \mathbf{y}
\end{aligned}
$$

and

$$
\begin{aligned}
& \lim _{\xi \rightarrow 0} \mathbf{H}^{-1} \\
& =\left(\mathbf{I}-<\boldsymbol{\kappa}>_{\mathbf{q}^{* *}(\boldsymbol{\kappa})}\left(\operatorname{Adiag}\left(<\mathbf{b}>_{\mathbf{q}^{* *}(\mathbf{b})}\right)<\boldsymbol{\kappa}>_{\mathbf{q}^{* *}(\boldsymbol{\kappa})}\right)^{+}\right) \\
& \times \operatorname{Adiag}\left(<\mathbf{b}>_{q^{* *}(\mathbf{b})}\right)<\boldsymbol{\kappa}>_{q^{* *}(\boldsymbol{\kappa})}^{2}
\end{aligned}
$$

\section{B. posterior approximation of $\mathbf{\Sigma}$}

As discussed in section $\Pi$, we have:

$$
\begin{gathered}
\ln \left(q^{*}(\boldsymbol{\Sigma})\right)=\ln \left(q^{*}\left(\boldsymbol{\kappa}^{2} \circ \boldsymbol{\gamma}^{2}\right)\right)=\ln \left(q^{*}\left(\boldsymbol{\kappa}^{2}\right)\right)+\ln \left(q^{*}\left(\boldsymbol{\gamma}^{2}\right)\right) \\
\propto<\ln P\left(\mathbf{a}, \mathbf{b}, \boldsymbol{\kappa}^{2} \circ \boldsymbol{\gamma}^{2} \mid \mathbf{y} ; \boldsymbol{\alpha}, \boldsymbol{\beta}\right)>_{q^{* *}}(\mathbf{b}), q^{* *}(\mathbf{a})
\end{gathered}
$$

Substituting (4), (5), (6) into (9) and using the separability of $\ln \left(q^{*}\left(\boldsymbol{\kappa}^{2}\right)\right)$, it can be shown that:

$$
\begin{aligned}
\ln q\left(\kappa_{i}^{2}\right) \propto-\frac{1}{2}( & \left.<a_{i}^{2}>_{q^{* *}(\mathbf{a})}+2 \beta<\gamma_{i}{ }^{-2}>_{q^{*}\left(\gamma_{i}^{2}\right)}\right) \kappa_{i}{ }^{-2} \\
& +\left(\alpha+\frac{1}{2}-1\right) \ln \left(\kappa_{i}^{-2}\right)
\end{aligned}
$$

which means

$$
\kappa_{i}^{-2} \sim \Gamma\left(\frac{1}{2}+\alpha, \frac{1}{2}\left(<a_{i}^{2}>_{q^{* *}(\mathbf{a})}+2 \beta<\gamma_{i}^{-2}>_{q^{*}\left(\boldsymbol{\gamma}^{2}\right)}\right)\right)
$$

and

$$
<\kappa_{i}{ }^{-2}>_{q^{*}\left(\boldsymbol{\kappa}^{2}\right)}=\frac{\frac{1}{2}+\alpha}{\frac{1}{2}\left(<a_{i}^{2}>_{q^{* *}(\mathbf{a})}+2 \beta<\gamma_{i}^{-2}>_{q^{*}\left(\boldsymbol{\gamma}^{2}\right)}\right)}
$$

Similarly, we have:

$$
\gamma_{i}^{-2} \sim \Gamma\left(\frac{1}{2}+\alpha, \frac{1}{2}\left(<b_{i}^{2}>_{q^{* *}(\mathbf{b})}+2 \beta<\kappa_{i}{ }^{-2}>_{q^{*}\left(\boldsymbol{\kappa}^{2}\right)}\right)\right)
$$

and

$$
<\gamma_{i}{ }^{-2}>_{q^{*}\left(\boldsymbol{\gamma}^{2}\right)}=\frac{\frac{1}{2}+\alpha}{\frac{1}{2}\left(<b_{i}^{2}>_{q^{* *}(\mathbf{b})}+2 \beta<\kappa_{i}{ }^{-2}>_{q^{*}\left(\boldsymbol{\kappa}^{2}\right)}\right)}
$$

\section{The Proposed Algorithm}

As a result, we summarize the procedure above as two algorithms named "NP-0" and "NP-1" which represent the inference results for the one layer signal model and two layer signal model respectively. The difference between $\mathrm{Np}-0$ and NP-1 is whether the learning process updates the precision of Normal Product.

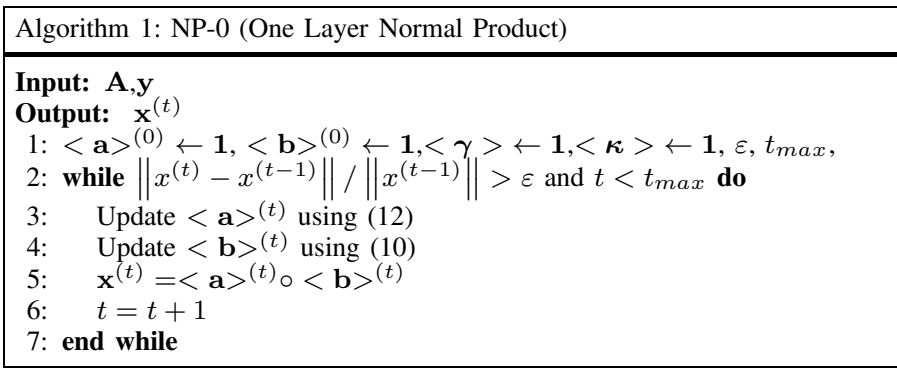

The algorithm NP-0 is similar to the FOCUSS algorithm [14]. The difference between them is that NP-0 uses $\langle\mathbf{a}\rangle^{(t)}$ and $<\mathbf{b}>^{(t)}$, the decomposed component of $\mathbf{x}^{(t)}$ in an interleaved way to update $\mathbf{x}^{(t+1)}$ while FOCUSS uses the whole $\mathbf{x}^{(t)}$ to directly update $\mathbf{x}^{(t+1)}$. Furthermore, we set the initial value of the algorithm as a constant to avoid the local minimum results being returned.

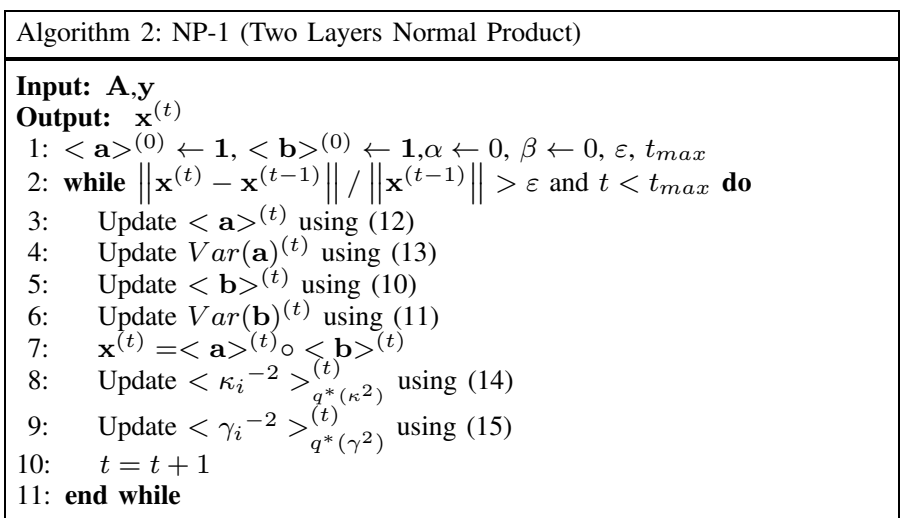

The algorithm NP-1 looks like coupling two sparse Bayesian learning (SBL) [6], [8] procedures together and we will experientially prove that the MSE via NP-1 descends faster than SBL in section IV 


\section{NumERICAL RESULTS}

In this section, we compare our proposed algorithms among SBL, iterative reweighted least squares (IRLS) [15] and basis pursuit (BP) [4]. In the following results, we set the dimension of original signal to be 100 and in each experiment every entries of the sensing matrix $\mathbf{A}$ is uniformly distributed as standard normal random variable. The stop criterion parameters $\varepsilon$ and $t_{\text {max }}$ in both algorithm NP-0 and NP-1 are set as $10^{-3}$ and 300 respectively.

The success rate in the Fig. 3 is defined by the average probability of successful restoration, which is counted as the ratio between the number of successful trials and the total number of the independent experiments. It collects the result of 300 Monte Carlo trials and we assume successful trial if $\|\hat{\mathbf{x}}-\mathbf{x}\|_{2} /\|\mathbf{x}\|_{2}<10^{-3}$, where $\hat{\mathbf{x}}$ is the estimate of the original signal $\mathbf{x}$. The mean squared error (MSE) in Fig. 4 which measures the average of the squares of the difference between the estimator and estimated signal is calculated as follow:

$$
M S E(d B)=20 \log \left(\|\hat{\mathbf{x}}-\mathbf{x}\|_{2} /\|\mathbf{x}\|_{2}\right)
$$

Fig. 3 demonstrats the superior performance of the NP-1 algorithm in a few measurements. It shows the success rate of respective algorithms versus the number of measurement $\mathrm{M}$ and sparse level $\mathrm{K}$ of the signal. We can see that the reconstruction performance of the one layer normal product algorithm(NP-0) is almost the same as BP, which is the MAP estimation of unknowns in the one layer Laplace prior framework. The comparisons also show that the two layers normal product algorithm(NP-1) requires as few number of $\mathrm{CS}$ measurements as SBL while inheriting the similar reconstruction precision.

In Fig. 4, the comparison among the computational cost of the three algorithms on the reconstruction performance are performed on a personal PC with dual-core $3.1 \mathrm{GHz} \mathrm{CPU}$ and $4 \mathrm{~GB}$ RAM. It is interesting to note that the MSE of NP-1 descends faster than SBL in Fig. 4(a) while it has the same sparsity-undersampling tradeoff performance as SBL. It should also be noticed that we haven't use any basis pruning technology as in [16] to reduce the computational complexity. To understand the reason of low computational time from Fig. 4(b), we observe that the NP-1 requires only a few numbers of iterations to arrive at stop condition. In summary, these experimental results confirm that the NP-1 is a fast Sparse Bayesian algorithm.

\section{CONCUlsion}

In this letter, we formulated a Normal Product prior based Bayesian framework to solve the compressed sensing problem in noise free case. Using this framework, we derived two algorithms named NP-0 and NP-1 and compared them with different algorithms. We have shown that our algorithm NP-1 has the similar reconstruction performance with SBL while the interleaved updating procedure provides improved performance in computational times.

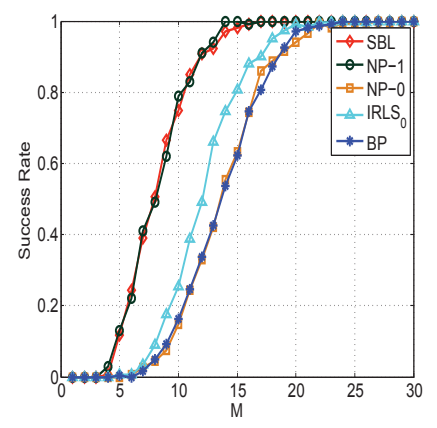

(a) $\mathrm{K}=3$

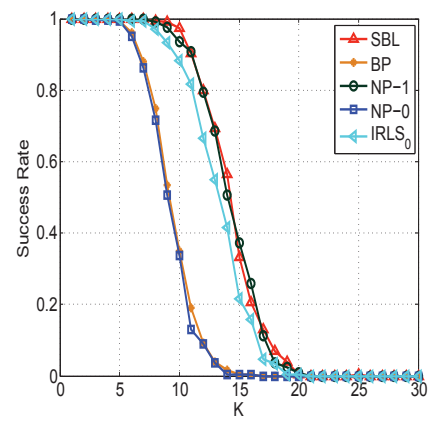

(b) $\mathrm{M}=30$
Fig. 3. Simulation results of Success Rate

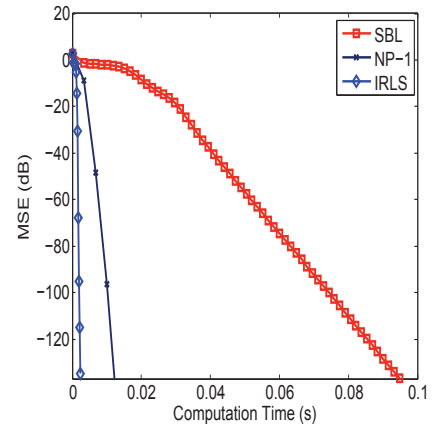

(a) $\mathrm{K}=3, \mathrm{M}=30$

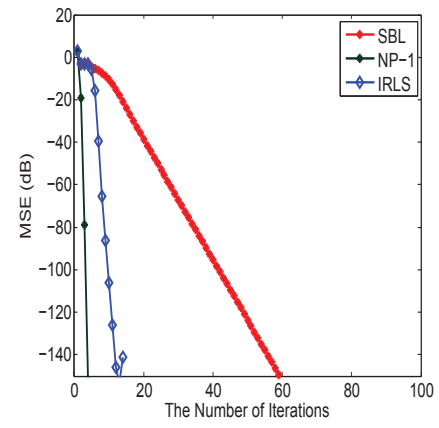

(b) $\mathrm{K}=3, \mathrm{M}=30$
Fig. 4. Simulation results of Computational Cost

\section{REFERENCES}

[1] D. L. Donoho, "Compressed sensing," IEEE Trans. Inf. Theory, vol. 52, no. 4, pp. 1289-1306, 2006.

[2] E. J. Candès, J. Romberg, and T. Tao, "Robust uncertainty principles: Exact signal reconstruction from highly incomplete frequency information,” IEEE Tran. Inf. Theory, vol. 52, no. 2, pp. 489-509, 2006.

[3] J. A. Tropp and A. C. Gilbert, "Signal recovery from random measurements via orthogonal matching pursuit," IEEE Trans. Inf. Theory, vol. 53, no. 12, pp. 4655-4666, 2007.

[4] S. S. Chen, D. L. Donoho, and M. A. Saunders, "Atomic decomposition by basis pursuit," SIAM J. Sci. Comput., vol. 20, no. 1, pp. 33-61, 1998.

[5] R. Chartrand, "Exact reconstruction of sparse signals via nonconvex minimization," IEEE Signal Process. Lett., vol. 14, no. 10, pp. $707-$ $710,2007$.

[6] M. E. Tipping, "Sparse bayesian learning and the relevance vector machine," J. Mach. Learn. Res., vol. 1, pp. 211-244, 2001.

[7] S. Ji, Y. Xue, and L. Carin, "Bayesian compressive sensing," IEEE Trans. Signal Process., vol. 56, no. 6, pp. 2346-2356, 2008.

[8] D. P. Wipf and B. D. Rao, "Sparse bayesian learning for basis selection," IEEE Trans. Signal Process., vol. 52, no. 8, pp. 2153-2164, 2004.

[9] S. D. Babacan, R. Molina, and A. K. Katsaggelos, "Bayesian compressive sensing using laplace priors," IEEE Trans. Image Process., vol. 19, no. 1, pp. 53-63, 2010 .

[10] E. W. Weisstein. Normal product distribution. MathWorldA Wolfram Web Resource. [Online]. Available: http://mathworld.wolfram.com/NormalProductDistribution.html

[11] A. SEIJAS-MACIAS and A. OLIVEIRA, "An approach to distribution of the product of two normal variables." Discussiones Mathematicae: Probability \& Statistics, vol. 32, 2012.

[12] D. Wipf, J. Palmer, and B. Rao, "Perspectives on sparse bayesian learning," Computer Engineering, vol. 16, no. 1, p. 249, 2004.

[13] D. G. Tzikas, C. Likas, and N. P. Galatsanos, "The variational approximation for bayesian inference," IEEE Signal Process. Mag., vol. 25 , no. 6, pp. 131-146, 2008. 
[14] I. F. Gorodnitsky and B. D. Rao, "Sparse signal reconstruction from limited data using focuss: A re-weighted minimum norm algorithm," IEEE Trans. Signal Process., vol. 45, no. 3, pp. 600-616, 1997.

[15] R. Chartrand and W. Yin, "Iteratively reweighted algorithms for compressive sensing," in Proc. ICASSP, 2008, pp. 3869-3872.

[16] M. E. Tipping, A. C. Faul et al., "Fast marginal likelihood maximisation for sparse bayesian models," in Proc. 9th Int. Workshop on AIStats, vol. 1, no. 3, 2003. 